\title{
COMPACTNESS OF THE $\bar{\partial}$-NEUMANN OPERATOR
}

\author{
STEVEN G. KRANTZ
}

(Communicated by John B. Conway)

\begin{abstract}
This paper gives an elementary construction of smooth, bounded pseudoconvex domains which satisfy Condition $\mathrm{R}$ but which do not have compact $\bar{\partial}$-Neumann operator.
\end{abstract}

0. Introduction. Several sufficient conditions are known for the $\bar{\partial}$-Neumann operator to be compact in the $L^{2}$ topology of a pseudoconvex domain in $\mathbf{C}^{n}$ (see [FK] for terminology). A global subelliptic estimate suffices (see, for instance, [FK, CA1]), as does the more general Condition $\mathrm{P}$ of Catlin [CA2].

In this note we exhibit a family of domains in which compactness of the Neumann operator in the Euclidean metric does not hold. Our calculations are notable for their elementary and explicit nature and for their behavior vis à vis Bell's Condition $\mathrm{R}$ [BE]. These connections will be discussed below.

I would like to thank Harold Boas and Norberto Salinas for useful conversations about this problem.

1. Definitions and main results. If $\Omega \subseteq \mathbf{C}^{n}$ is a domain (a connected open set), we say that $\Omega$ is Reinhardt if whenever $\left(z_{1}, \ldots, z_{n}\right) \in \Omega$ then $\left(e^{i \theta_{1}} z_{1}, \ldots, e^{i \theta_{n}} z_{n}\right)$ $\in \Omega$ for any real $\theta_{1}, \ldots, \theta_{n}$.

We say that $\Omega$ has a flat boundary neighborhood if the following conditions hold.

(i) $\Omega$ is contained in a polydisc $\mathscr{D}=D\left(0, r_{1}\right) \times \cdots \times D\left(0, r_{n}\right)$.

(ii) There is a point $P \in \partial \mathscr{D}, P$ not in the distinguished boundary $\partial D\left(0, r_{1}\right) \times$ $\cdots \times \partial D\left(0, r_{n}\right)$ of $\mathscr{D}$, and a neighborhood $U \subseteq \mathbf{C}^{n}$ of $P$ such that $U \cap \partial \Omega=U \cap \partial \mathscr{D}$.

To understand the meaning of "flat boundary neighborhood," take $\Omega \subseteq \mathbf{C}^{2}$, $\mathscr{D}=D(0,1) \times D(0,1)$, and $P=(1, q)$ for some fixed $q \in \mathbf{C}$ with $|q|<1$. If $\Omega$ has a flat boundary neighborhood at $P$ (relative to $\mathscr{D}$ ), then for $\varepsilon>0$ small there is a

$$
U_{\varepsilon}=\left\{\left(z_{1}, z_{2}\right):\left|z_{1}-1\right|<\varepsilon,\left|z_{2}-q\right|<\varepsilon\right\}
$$

such that $U_{\varepsilon} \cap \partial \Omega=U_{\varepsilon} \cap \partial \mathscr{D}$. If, in addition, $\Omega$ is Reinhardt, then $\Omega$ contains the region $(D(0,1) \backslash \bar{D}(0,1-\varepsilon)) \times D(q, \varepsilon)$, and $\partial \Omega$ contains $\left\{e^{i \theta}\right\} \times D(q, \varepsilon)$. In particular, if $\Omega$ is a polydisc with "rounded corners," then $\Omega$ has a flat boundary neighborhood.

THEOREM. If $\Omega \subseteq \mathbf{C}^{n}$ is a bounded, smooth Reinhardt domain with a flat boundary neighborhood then the $\bar{\partial}-$ Neumann operator in the Euclidean metric on $(0,1)$ forms is not compact in the $L^{2}$ topology of $\Omega$.

Received by the editors July 23, 1987.

1980 Mathematics Subject Classification (1985 Revision). Primary 35N15; Secondary 32F20.

Key words and phrases. Cauchy-Riemann equations, Neumann operator, pseudoconvex domain. Supported in part by the National Science Foundation. 
REMARK 1. Catlin (private communication) has proved that a smooth pseudoconvex domain in $\mathbf{C}^{2}$ with an analytic disc in the boundary has noncompact $\bar{\partial}$-Neumann operator. Domains of this kind do not satisfy Condition P of [CA2].

REMARK 2. Bell and Boas [BB] have shown that if $\Omega$ is complete Reinhardt and smooth, then $\Omega$ satisfies Condition $R$. Thus the theorem provides us with easy examples of domains satisfying Condition $\mathrm{R}$ yet with noncompact $\bar{\partial}$-Neumann operator. We also mention the very nice [HPB] which contains related results.

REMARK 3. N. Salinas has constructed a proof (private communication) that the $\bar{\partial}$-Neumann operator of the bidisc is noncompact. He uses rather sophisticated $C^{*}$ algebra techniques which only apply to a product domain.

2. Proof of the Theorem. For simplicity we give the proof on the bidisc and indicate afterward the necessary modifications for the general result.

Let

$$
D=\{\varsigma \in \mathbf{C}:|\varsigma|<1\} \text { and } \varphi_{j}(\varsigma)=\sqrt{\frac{j+1}{\pi}} \varsigma^{j}
$$

Then

$$
\left\|\varphi_{j}\right\|_{L^{2}(D)}=1, \quad \text { all } j=1,2, \ldots
$$

Define forms

$$
\alpha_{j}\left(z_{1}, z_{2}\right)=\varphi_{j}\left(z_{1}\right) d \bar{z}_{2} .
$$

Then each $\alpha_{j}$ is a $\bar{\partial}$-closed $(0,1)$ form and

$$
\left\|\alpha_{j}\right\|_{L^{2}(D \times D)}=\sqrt{\pi} \text {. }
$$

The canonical solution (i.e., the solution orthogonal to holomorphic functions) to $\bar{\partial} u=\alpha_{j}$ is

$$
u_{j}\left(z_{1}, z_{2}\right)=\varphi_{j}\left(z_{1}\right) \bar{z}_{2}
$$

For surely $\bar{\partial} u_{j}=\alpha_{j}$. Also if $h$ is $L^{2}$ and holomorphic on $D \times D$, then

$$
\begin{aligned}
& \int u_{j}\left(z_{1}, z_{2}\right) \overline{h\left(z_{1}, z_{2}\right)} d V\left(z_{1}, z_{2}\right) \\
& \quad=c \int_{D} \varphi_{j}\left(z_{1}\right) \int_{D} \bar{z}_{2} \overline{h\left(z_{1}, z_{2}\right)} d \bar{z}_{2} \wedge d z_{2} \wedge d \bar{z}_{1} \wedge d z_{1} \\
& \quad=0
\end{aligned}
$$

by the mean value property, since $D \times D$ is circular in $z_{2}$.

Finally,

$$
\left\|u_{j}\right\|_{L^{2}(D \times D)}=\sqrt{\pi / 2} \text { and } u_{j} \perp u_{k} \text { if } j \neq k
$$

since $D \times D$ is circular in $z_{1}$. Thus $\left\{u_{j}\right\}$ has no convergent subsequence in $L^{2}(D \times D)$. This shows that $\bar{\partial}^{*} N$ is not a compact operator.

Now an easy calculation shows that

$$
N \alpha_{j}=\left|z_{2}\right|^{2} \varphi_{j}\left(z_{1}\right) d \bar{z}_{2} \equiv \rho_{j} .
$$

(Since the harmonic space is void, and $\bar{\partial} \alpha_{j}=0$, we need only check that $\bar{\partial}^{*} \rho_{j}=u_{j}$.) As before, we have $\left\|N \alpha_{j}\right\|_{L^{2}} \geq K_{0}>0$ and the $N \alpha_{j}$ are orthogonal. So $N$ is not compact.

REMARK 4. It is interesting to observe that on domains of the form

$$
U=\left\{z:\left|z_{1}\right|^{2}+\left|z_{2}\right|^{2 m}<1\right\}, \quad m \in\{1,2, \ldots\}
$$


or even on a domain with an infinitely flat spot, such as

$$
U^{\prime}=\left\{z:\left|z_{1}\right|^{2}+e^{-1 /\left|z_{2}\right|^{2}}<1 / 2\right\}
$$

the forms $\alpha_{j}$ and $\bar{\partial}$-solutions $u_{j}$ still make sense. Moreover, $\left\|u_{j}\right\|_{L^{2}}$ is uniformly bounded, as is $\left\|\alpha_{j}\right\|_{L^{2}}$. Finally, the $u_{j}$ are pairwise orthogonal. However, for either $U$ or $U^{\prime}$, because of the curvature of the boundary at points $\left(e^{i \theta}, 0\right)$, it holds that $\left\|u_{j}\right\|_{L^{2}} \rightarrow 0$ as $j \rightarrow \infty$. So no counterexample to compactness results. (And, on both $U$ and $U^{\prime}$, it is known that $N$ and $\bar{\partial}^{*} N$ are compact because their domains satisfy Catlin's Condition P.)

Finally, it is easy to see how our proof for the polydisc can be adapted to get the full result of the theorem. For such a domain $\Omega$, we have (as in the remarks preceding the statement of the theorem) after permuting coordinates that

$$
\begin{aligned}
\mathscr{D} & \equiv D\left(0, r_{1}\right) \times \cdots \times D\left(0, r_{n}\right) \supseteq \Omega \\
& \supseteq\left(D\left(0, r_{1}\right) \backslash \bar{D}\left(0, r_{1} \backslash \varepsilon\right)\right) \times D\left(P_{2}, \varepsilon\right) \times \cdots \times D\left(P_{n}, \varepsilon\right) \equiv \mathscr{E}
\end{aligned}
$$

and

$$
\partial \Omega \supseteq\left\{\left(r_{1}, e^{i \theta}\right)\right\} \times D\left(P_{2}, \varepsilon\right) \times \cdots \times D\left(P_{n}, \varepsilon\right) .
$$

The forms $\left\{z_{1}^{j} d \bar{z}_{2}\right\}$ and functions $\left\{z_{1}^{j} \bar{z}_{2}\right\}$, when suitably normalized, will be uniformly bounded in $L^{2}$ (by comparison with their norms on $\mathscr{D}$ ) and bounded in norm from below by their norms on $\mathscr{E}$. They will be orthogonal since $\Omega$ is Reinhardt. Thus, as before, we arrive at a counterexample to compactness.

\section{REFERENCES}

[BE] S. Bell, Biholomorphic mappings and the $\bar{\partial}$ problem, Ann. of Math. (2) 114 (1981), 103-113.

[BB] S. Bell and H. Boas, Regularities of the Bergman projection in weakly pseudoconvex domains, Math. Ann. 257 (1981), 23-30.

[HPB] H. P. Boas, Small sets of infinite type are benign for the $\bar{\partial}$-Neumann problem, preprint.

[CA1] D. Catlin, Subelliptic estimates for the $\bar{\partial}$-Neumann problem on pseudoconvex domains, Ann. of Math. (in press).

[CA2] - Global regularity of the $\bar{\partial}-$ Neumann problem, Proc. Sympos. Pure Math., vol. 41, Amer. Math. Soc., Providence, R. I., 1984.

[FK] G. B. Folland and J. J. Kohn, The Neumann problem for the Cauchy-Riemann complex, Princeton Univ. Press, Princeton, N. J., 1972.

Department of Mathematics, Washington University, St. Louis, Missouri 63130 